\title{
A Critical Role of Nitric Oxide in Human Immunodeficiency Virus Type 1-Induced Hyperresponsiveness of Cultured Monocytes
}

\author{
Michael Bukrinsky, * Helena Schmidtmayerova, ${ }^{\dagger}$ \\ Gabriele Zybarth,* Larisa Dubrovsky,* Barbara Sherry,* \\ and Grigori Enikolopov \\ *The Picower Institute for Medical Research, Manhasset, New York, \\ U.S.A. \\ 'Institute of Virology, Slovak Academy of Sciences, Slovakia \\ ${ }^{\ddagger}$ Cold Spring Harbor Laboratory, Cold Spring Harbor, New York, \\ U.S.A.
}

\begin{abstract}
Background: Human immunodeficiency virus type 1 (HIV-1) infection leads to a general exhaustion of the immune system. Prior to this widespread decline of immune functions, however, there is an evident hyperactivation of the monocyte/macrophage arm. Increased levels of cytokines and other biologically active molecules produced by activated monocytes may contribute to the pathogenesis of HIV disease both by activating expression of HIV-1 provirus and by direct effects on cytokine-sensitive tissues, such as lung or brain. In this article, we investigate mechanisms of hyperresponsiveness of HIV-infected monocytes.

Materials and Methods: The study was performed on monocyte cultures infected in vitro with a monocytetropic strain HIV-1 $1_{\mathrm{ADA}}$. Cytokine production was induced by stimulation of cultures with lipopolysaccharides (LPS) and measured by ELISA. To study involvement of nitric oxide $(\mathrm{NO})$ in the regulation of
\end{abstract}

cytokine expression, inhibitors of nitric oxide synthase (NOS) or chemical donors of NO were used.

Results: We demonstrate that infection with HIV-1 in vitro primes human monocytes for subsequent activation with LPS, resulting in increased production of proinflammatory cytokines tumor necrosis factor (TNF) and interleukin 6 (IL-6). This priming effect can be blocked by $\mathrm{Ca}^{2+}$-chelating agents and by the NOS inhibitor LNMMA, but not by hemoglobin. It could be reproduced on uninfected monocyte cultures by using donors of NO, but not cGMP, together with LPS.

Conclusions: NO, which is expressed in HIV-1-infected monocyte cultures, induces hyperresponsiveness of monocytes by synergizing with calcium signals activated in response to LPS stimulation. This activation is cGMP independent. Our findings demonstrate the critical role of NO in HIV-1-specific hyperactivation of monocytes.

\section{INTRODUCTION}

Acquired immunodeficiency syndrome (AIDS) patients often show elevated circulating levels of pro-inflammatory monocyte-produced cytokines (monokines), particularly tumor necrosis factor (TNF) and interleukin 6 (IL-6) $(1,2)$. These monokines, produced as a result of cell activa-

Address correspondence and reprint requests to: Michael Bukrinsky, The Picower Institute for Medical Research, 350 Community Drive, Manhasset, NY 11030, U.S.A. tion, can be expected to stimulate nearby uninfected cells, thus contributing to a generalized activation of the monocyte/macrophage arm of the immune system. In addition, pro-inflammatory cytokines have been shown to be powerful activators of human immunodeficiency virus type 1 (HIV-1) replication (3-5). Such abnormal activation is likely one of the important pathogenic factors in the development of AIDS $(6,7)$. However, pathophysiological mechanisms that lead to enhanced cytokine production in AIDS 
remain unclear. Phenomenon of monocyte hyperactivation can be reproduced in vitro. Monocyte cultures infected in vitro with a monocytotropic HIV-l strain produce significantly higher levels of pro-inflammatory monokines in response to stimulation than do similarly stimulated uninfected cultures $(8,9)$, making such cultures a good model for studying events that lead to monokine hyperproduction in AIDS.

Recently, we demonstrated both induction of nitric oxide synthase (NOS) in and production of nitric oxide (NO) by HIV-1-infected monocytes (10). NO is a well-recognized mediator of cell activity, being involved in the regulation of various cellular functions (reviewed in Ref. 11), including gene expression (12-14). Many of the known inductive effects of NO have been attributed to its ability to activate guanylate cyclase and generate cGMP (15). Recent reports, however, suggest that NO may activate human peripheral blood monocytes (PBMC) $(12,13,16)$ in a cGMP-independent way (13) and that it could be involved in the regulation of cytokine production by monocytes. We, therefore, examined whether NO produced by HIV-infected monocyte cultures is responsible for their hyperresponsiveness to stimulation. In this report, we demonstrate that NO enhances monocyte responses to stimulation by synergizing with $\mathrm{Ca}^{2+}$ signals. This effect is cGMP independent and most likely exerted in an autocrine fashion.

\section{MATERIALS AND METHODS}

\section{Chemicals}

NO generators SNAP and NONO were purchased from Toronto Research Chemicals Inc. (Downsview, Ontario, Canada); guanylate cyclase inhibitor LY83583 was obtained from Research Biochemicals Inc. (Natick, MA, U.S.A.); other chemicals were from Sigma Chemical Co. (St. Louis, MO, U.S.A.).

\section{Monocyte Cultures}

Human monocytes were isolated from blood of healthy donors negative for HIV and hepatitis B antibodies by adherence to plastic as follows. Concentrated blood was diluted six times with Dulbecco's Modified Eagle's medium (DMEM, Sigma) and loaded on a Ficoll-Paque (Pharmacia Biotech, Uppsala, Sweden) gradient. After a 20min centrifugation at $200 \times g$ at room tempera- ture, the middle layer (PBMCs) was transferred to a new tube and gently washed three times with DMEM. Cells were then resuspended in DMEM supplemented with $10 \%$ heat-inactivated pooled human serum and $1 \%$ penicillin/ streptomycin (Sigma) and counted. Cell suspension diluted to $5-8 \times 10^{6} \mathrm{cells} / \mathrm{ml}$ was placed in Primaria (Becton Dickinson, Franklin Lakes, NJ, U.S.A.) flasks and incubated $2 \mathrm{hr}$ at $37^{\circ} \mathrm{C}$. Adherent cells were washed three times with DMEM and left overnight at $37^{\circ} \mathrm{C}$ in DMEM supplemented with $10 \%$ human serum and $1 \%$ penicillin/streptomycin. Next day, cells were washed with cold phosphate-buffered saline (PBS, Sigma), incubated 3-5 min with cold 10 mM EDTA solution in PBS, and detached with a cell scraper. At that step, cells were counted, resuspended in DMEM $+10 \%$ human serum + $1 \%$ penicillin/streptomycin $+1 \mathrm{ng} / \mathrm{ml} \mathrm{M}$-CSF (Sigma) at $1 \times 10^{6}$ cells $/ \mathrm{ml}$, and placed into 24-well Primaria plates. Cells were allowed to differentiate for 7 days (half of the medium was changed on Day 3 and 5 after isolation) in the presence of M-CSF. Subsequent incubations for infection with HIV-1, cell stimulation, etc., were performed in the medium without M-CSF. Monocyte cultures prepared by this method were consistently $>96 \%$ pure monocytes by the criteria of cell morphology on Wright-stained cytosmears and by nonspecific esterase assays.

\section{Infection with HIV-1}

Adherent monocytes cultured for 7 days were exposed to a monocytotropic viral strain HIV$1_{\mathrm{ADA}}$ at a concentration corresponding to reverse transcriptase (RT) activity of $2 \times 10^{5} \mathrm{cpm} / \mathrm{ml}$. After an overnight incubation at $37^{\circ} \mathrm{C}$, excess of the virus was washed away, and incubation was continued for 2-3 weeks. Culture medium was half-exchanged every 2-3 days.

\section{RT Assay}

Reaction mixture $(50 \mu \mathrm{l})$ containing $10 \mu \mathrm{l}$ of supernatant from HIV-infected culture (precleared from cell debris by centrifugation at $5000 \times g$ for $5 \mathrm{~min}), 50 \mathrm{mM}$ Tris $(\mathrm{pH} \mathrm{7.8}), 20$ $\mathrm{mM} \mathrm{KCl}, 5 \mathrm{mM} \mathrm{MgCl}$, $1 \mathrm{mM}$ DTT, $0.2 \mathrm{OD} / \mathrm{ml}$ poly(A), $0.2 \mathrm{OD} / \mathrm{ml}$ oligo(dT) $12-18,0.1 \%$ Triton $\mathrm{X}-100$, and $40 \mu \mathrm{Ci} / \mathrm{ml}^{3} \mathrm{H}$-TTP (76 Ci/mmol; DuPont, Wilmington, DE, U.S.A.), was incubated 2 $\mathrm{hr}$ at $37^{\circ} \mathrm{C}$. Five microliters of the reaction mixture were then spotted onto the DE81 (Whatman, Hillsboro, OR, U.S.A.) ion exchange paper, 
air dried, and washed five times with $5 \%$ $\mathrm{Na}_{2} \mathrm{HPO}_{4}$, followed by rinsing with distilled water. Air-dried filters were covered with scintillation fluid in Flexi Filter plate (Packard, Downers Grove, IL, U.S.A.) and counted in a Top Count Microplate Counter (Packard). Results are expressed as counts per minute per $1 \mathrm{ml}$ of culture supernatant $(\mathrm{cpm} / \mathrm{ml})$.

\section{Cell Stimulation}

HIV-1-infected cultures were incubated until RT activity in the culture supernatants reached $0.7-$ $1.1 \times 10^{6} \mathrm{cpm} / \mathrm{ml} \mathrm{(6-10} \mathrm{days).} \mathrm{Replicate} \mathrm{cul-}$ tures of control (uninfected) cells were maintained under similar conditions. Treatments were performed in three independent wells for each combination of agents. Usually, all compounds were added simultaneously at the time when RT reached an indicated level and were present throughout the duration of an experiment $(24-48 \mathrm{hr})$.

\section{Cytokine Assays}

TNF and IL- 6 in culture supernatants were determined by Medgenix EASIA System (Incstar Corp., Stillwater, MN, U.S.A.) utilizing non-neutralizing monoclonal antibodies in the development of immunoassay and thus allowing detection of both free and soluble receptor-bound cytokines.

\section{RESULTS}

\section{Inhibitors of NOS Reduce Amounts of Pro-Inflammatory Cytokines Produced by LPS-Stimulated HIV-1-Infected Monocyte Cultures}

Analysis of supernatants from monocyte cultures infected in vitro with the monocytotropic strain HIV- $1_{\text {ADA }}$ did not reveal induction of TNF or IL- 6 (not shown). HIV-infected monocytes stimulated with LPS, however, produced significantly higher levels of TNF (Fig. 1) and other pro-inflammatory cytokines (not shown) than similarly stimulated uninfected controls, in agreement with recently reported results $(8,9)$. This HIV-specific enhancement of cytokine production was observed during the entire time course of the LPS-induced effect, lasting 24-36 hr (9) (Fig. 1D). In accordance with previously published results (9), however, the greatest difference between HIV-infected and uninfected cul- tures was seen $18-24 \mathrm{hr}$ after the LPS stimulation. This time point was thus chosen for further analysis. Although there was a substantial degree of variation in the absolute values of expressed cytokines in monocyte cultures prepared from different donors (compare TNF values in Fig. $1 \mathrm{~A}-\mathrm{C}$ ), the HIV-1 infection-specific increases in cytokine production relative to uninfected (control) cultures were donor independent and were influenced primarily by the rate of HIV-1 infection (10). In these experiments, we used HIV-infected monocyte cultures at a time when RT activity in the culture supernatant reached about $10^{6} \mathrm{cpm} / \mathrm{ml}$ (5-7 days after infection), and most results reported here are presented as changes in cytokine production relative to control samples, designated as $100 \%$. The observed increase in cytokine levels after stimulation of HIV-infected cultures indicates that HIV-1 primes monocytes for a much stronger response to activating stimuli, thus resulting in a hyperactivated phenotype as soon as an appropriate second signal is received.

Recently, we have demonstrated that infection with HIV-1 induces expression of iNOS in human monocytes (10). To determine whether NO plays a role in establishing the hyperactivation phenotype in HIV-1-infected monocytes, we measured cytokine production in LPS-stimulated, HIV-infected monocyte cultures in the presence of NOS inhibitor L-NMMA. This inhibitor significantly suppressed the LPS-induced superproduction of TNF by HIV-infected monocytes, resulting in expression levels close to those observed in similarly stimulated uninfected cultures (Fig. 1 A-C). A similar effect was seen with other inhibitors of NOS L-NAME and aminoguanidine (results not shown). Inhibition was not observed with D-NMMA (Fig. 1), a noninhibitory analog of L-NMMA. NOS inhibitors did not affect levels of TNF produced by LPS-stimulated monocyte cultures (data not shown). This is in agreement with the previously demonstrated failure of LPS to induce NOS in human monocytes $(10,17)$ and argues against the recently suggested role for NO in LPS-mediated monocyte activation (16). Nitric oxide, therefore, seems to be one of the major factors that regulate overexpression of cytokines by HIV-infected monocytes.

\section{NO Donors Enhance LPS-Induced Production of TNF by Human Monocytes}

To prove the role of NO in monocyte hyperactivation, we used several chemical donors of NO. 

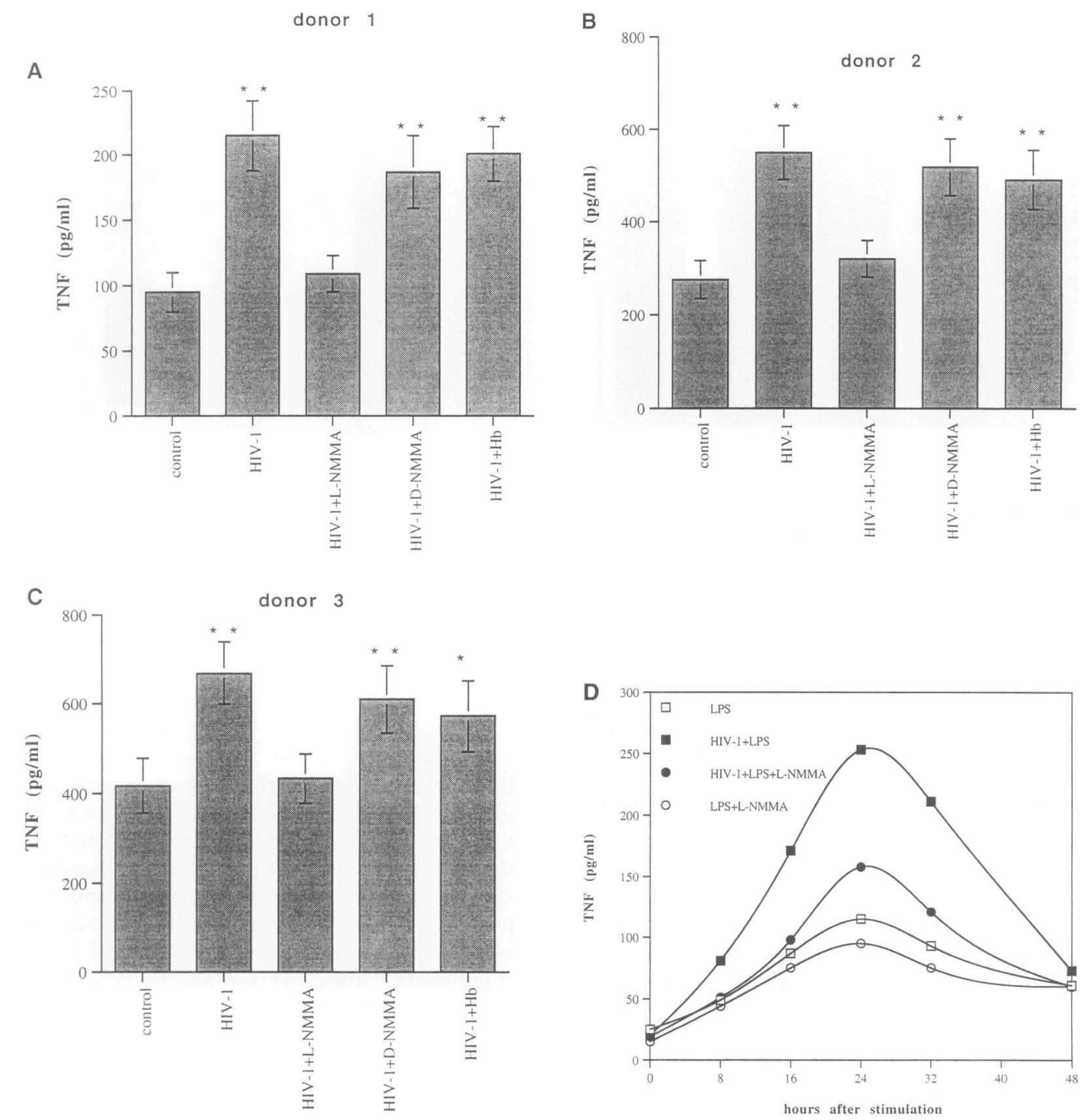

FIG. 1. Inhibition of nitric oxide synthase prevents superproduction of TNF by LPS-stimulated HIV-1-infected human monocytes

HIV-1-infected and uninfected (control) monocyte cultures prepared from peripheral blood of three seronegative donors were stimulated with $0.5 \mathrm{ng} / \mathrm{ml}$ of LPS alone or in combination with L-NMMA (2 mM), D-NMMA (2 mM), or hemoglobin $(\mathrm{Hb}, 100 \mu \mathrm{g} / \mathrm{ml})$. At the time of stimulation, RT values in culture supernatants were $0.6 \times 10^{6}$ $\mathrm{cpm} / \mathrm{ml}(\mathrm{A}$, donor 1$), 1.3 \times 10^{6} \mathrm{cpm} / \mathrm{ml}(\mathrm{B}$, donor 2$)$, and $1.1 \times 10^{6} \mathrm{cpm} / \mathrm{ml}(\mathrm{C}$, donor 3). Eighteen hours after stimulation, TNF in the culture supernatants was assayed by enzyme-linked immunosorbent assay (ELISA). For a time course experiment (D), HIV-infected and control monocyte cultures prepared from peripheral blood of donor 1 were stimulated with $0.5 \mathrm{ng} / \mathrm{ml}$ of LPS alone or in combination with L-NMMA ( $2 \mathrm{mM}$ ), and culture supernatants collected at 8,24 , and $48 \mathrm{hr}$ after infection were assayed by TNF-specific ELISA. Three independent wells for each treatment were analyzed, and the results are presented for each donor as mean \pm SD. Statistical analysis of the differences between L-NMMA-treated and untreated HIV-infected cultures was performed using a one-tailed $t$ test; ${ }^{*} p<0.05 ;{ }^{* *} p<0.01$. 


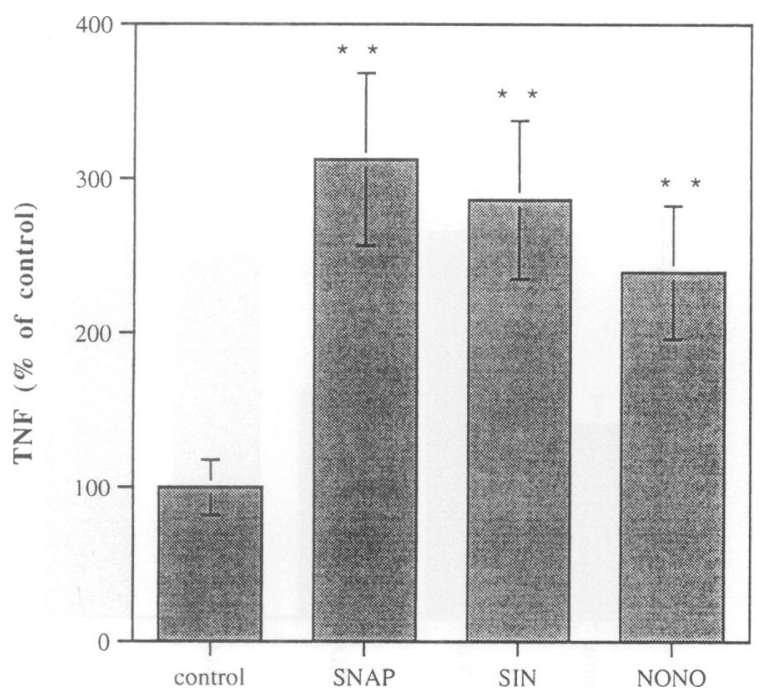

FIG. 2. Nitric oxide generators amplify LPS-induced expression of TNF

Replicate monocyte cultures were stimulated with $0.5 \mathrm{ng} / \mathrm{ml}$ LPS alone (control) or in combination with generators of NO SNAP $(100 \mu \mathrm{M})$, SIN (10 $\mu \mathrm{M})$, or NONO $(10 \mu \mathrm{M})$. Eighteen hours after stimulation, TNF levels in culture supernatants were assayed by ELISA. Values are scaled relative to LPSstimulated cultures taken as 100\%. Monocytes from two different donors were used, and the results of a representative experiment are presented. Results are shown as mean \pm SD of three separate wells. Statistical analysis was performed as in Fig. 1.

SNAP, SIN, and NONO significantly up-regulated production of TNF by uninfected monocyte cultures stimulated with LPS (Fig. 2). Similar results were obtained for IL-6 production (not shown). Without LPS stimulation, NO donors did not induce any measurable expression of cytokines. Although the NO donors used in this study can have some biological activities separate from NO production, the fact that we observed similar effects with chemically very different compounds argues against this explanation. This experiment does not prove that NO is the effector molecule in the process of up-regulation of cytokine expression, but together with reciprocal experiments using NOS inhibitors (Fig. 1) it argues for the pivotal role of NO in the induction of this effect.

Additional evidence for the role of $\mathrm{NO}$ in HIV-1-specific hyperactivation of monocytes was provided by reconstitution experiments. HIV-1infected monocytes were stimulated with LPS and cultured in the presence of NOS inhibitor L-NMMA and NO-generating compound SNAP.

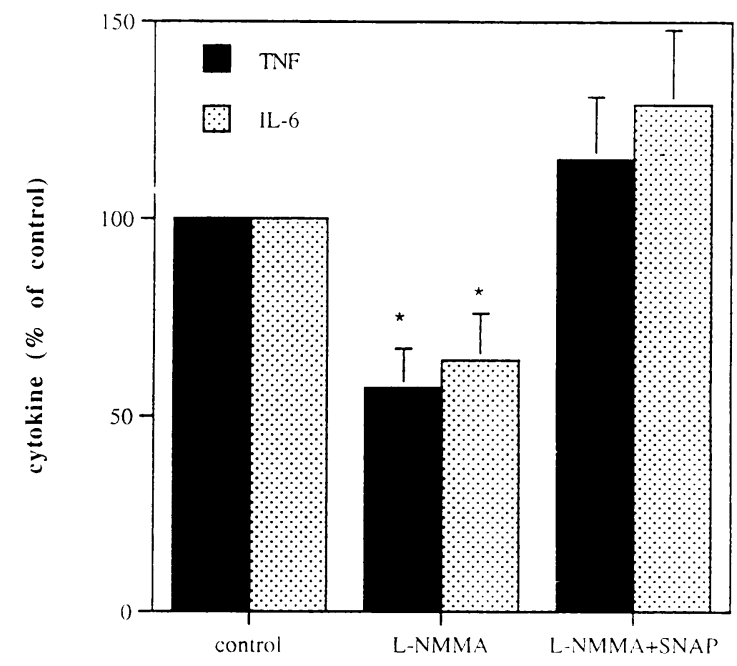

FIG. 3. NO cancels inhibitory effects of NOS inhibitor on cytokine production by HIV-1-infected LPS-stimulated monocytes

HIV-1-infected monocyte cultures were stimulated with $0.5 \mathrm{ng} / \mathrm{ml}$ LPS alone (control), in combination with L-NMMA (2 mM), or with L-NMMA ( $2 \mathrm{mM})$ and SNAP $(100 \mu \mathrm{M})$. Values are scaled relative to control $(100 \%)$. Error bars represent SD obtained from three replicates. Statistical analysis was performed as in Fig. 1.

Because L-NMMA reduces NO concentration by interacting with NOS, but not with NO, we anticipated that the NO-generator would reverse the inhibitory effect of L-NMMA on cytokine superproduction. Indeed, levels of TNF and IL- 6 produced by monocytes under these conditions were statistically similar to those observed in the supernatants from HIV-1-infected, LPS-stimulated monocyte cultures (Fig. 3).

\section{Autocrine Mechanism of NO Activity in HIV-Infected Monocytes}

$\mathrm{NO}$ is a potent local messenger molecule, capable of rapid migration from cell to cell, thus exerting its effects in both autocrine and paracrine fashion (18); the paracrine mode is, however, the major mechanism of NO effects (19). To determine its level of action in HIV-1-infected monocyte cultures, we used a high-molecular weight scavenger of NO, hemoglobin, which is not freely exchanged between the intra- and extracellular fluids and therefore mainly affects extracellular levels of NO (20). Hemoglobin did not have any significant effect on TNF production by HIV-1 infected, LPS-stimulated monocyte cultures (Fig. 1). Hemoglobin, however, decreased the 
activating effect of NO-generating compounds on LPS-stimulated monocytes (not shown), demonstrating its potency as an extracellular NO scavenger. This experiment suggests that HIV-1induced NO exerts its effects mostly in an autocrine fashion that does not depend on an extracellular phase. This explanation seems even more likely given a relatively low NO output by HIV-infected monocytes, thus increasing chances for its consumption within the producer cell. This result differs from the one reported by $\mathrm{Zi}$ netti et al. (16), who observed similar inhibition of TNF production by hemoglobin and by L-NMMA in LPS-stimulated mixed populations of PBMCs. We explain this difference by the contribution to NO production by non-monocytic cells (e.g., B lymphocytes [21]) in PBMCs, since we never observed NO effects in LPS-stimulated uninfected monocyte cultures. A possibility remains that other $\mathrm{NO}_{\mathrm{x}}$ species not scavenged by hemoglobin are produced intracellularly by HIV-infected monocytes in our experimental setting and that these are responsible for the observed effects. Although we cannot formally discount this possibility, the fact that HIV-1 produces the same effect on LPS-induced cytokine expression as addition of NO generators, and that the effect of NO generators can be blocked by hemoglobin, argues against this explanation.

\section{NO Synergizes with Calcium in the Induction of Cytokines}

NO can synergize with $\mathrm{Ca}^{2+}$ in the activation of immediate early genes in neuronal cells (14). Because LPS affects intracellular levels of $\mathrm{Ca}^{2+}$ $(22,23)$, we investigated the possibility that NO works in LPS-stimulated monocytes through a similar mechanism by amplifying the calcium signals. Monocyte cultures were treated with the following two compounds, which increase intracellular concentration of $\mathrm{Ca}^{2+}$ : thapsigargin, which releases $\mathrm{Ca}^{2+}$ from intracellular stores by inhibiting the endoplasmic reticulum $\mathrm{Ca}^{2+}$. ATPase (24), and a calcium ionophore, A23187. Both compounds induced TNF secretion by human monocytes (Fig. 4A). This effect was amplified when monocytes were either infected with HIV-1 or incubated in the presence of an NOgenerating compound, SNAP (Fig. 4A), thus resembling HIV-and NO-mediated amplification of LPS stimulation in identical cellular settings.

Although results presented above demonstrate that $\mathrm{NO}$ amplifies $\mathrm{Ca}^{2+}$-induced expres- sion of cytokines, they do not prove that calcium is involved in NO-mediated amplification of LPS stimulation of HIV-1-infected monocytes. To test this hypothesis, we stimulated HIV-1-infected and control monocyte cultures with LPS in the presence of the $\mathrm{Ca}^{2+}$ chelators, EGTA, which binds extracellular calcium, and BAPTA, which efficiently diffuses into the cell and reduces intracellular concentrations of $\mathrm{Ca}^{2+}$. Both agents reduced production of TNF by uninfected LPSstimulated monocytes by about $30 \%$ (Fig. 4B). The effect of the compounds on HIV-infected cultures was substantially greater, completely eliminating HIV-specific superproduction of TNF (Fig. 4B). Similar results were obtained with uninfected cultures stimulated with LPS in the presence of the NO generator SNAP. Both EGTA and BAPTA eliminated NO-specific amplification of TNF production by monocyte cultures (Fig. 4B). This experiment suggests that while $\mathrm{Ca}^{2+}$-mediated signaling is only a minor component of LPSspecific induction of cytokines in human monocytes (25), it is the major target for NO-regulated amplification.

\section{NO Activates Monocytes by a cGMP-Independent Mechanism}

Many of the known inductive effects of NO have been attributed to its ability to activate guanylate cyclase and generate cGMP (15). Therefore, we examined whether the cell pervious cGMP analogue, 8-Br-cGMP, could also amplify LPS-induced TNF production by human monocytes. No effect of 8-Br-cGMP at concentrations 10 and $100 \mu \mathrm{M}$ on TNF levels was observed (data not shown), thus suggesting a cGMP-independent mechanism of NO-mediated potentiation of LPS effect. This was further supported by experiments with LY-83583, an inhibitor of guanylate cyclase (26). When used at the $10 \mu \mathrm{M}$ concentration, this inhibitor did not diminish the NOmediated increase of LPS-induced TNF production in HIV-infected monocyte cultures (not shown).

\section{NO Produced in HIV-Infected Monocyte Cultures Does Not Provide Anti-Viral Defense}

Since NO was shown to have anti-viral activity $(27,28)$, including recently demonstrated inhibitory effect on a murine retrovirus (29), we investigated the possibility that NO production could be a defense reaction of monocytes to 

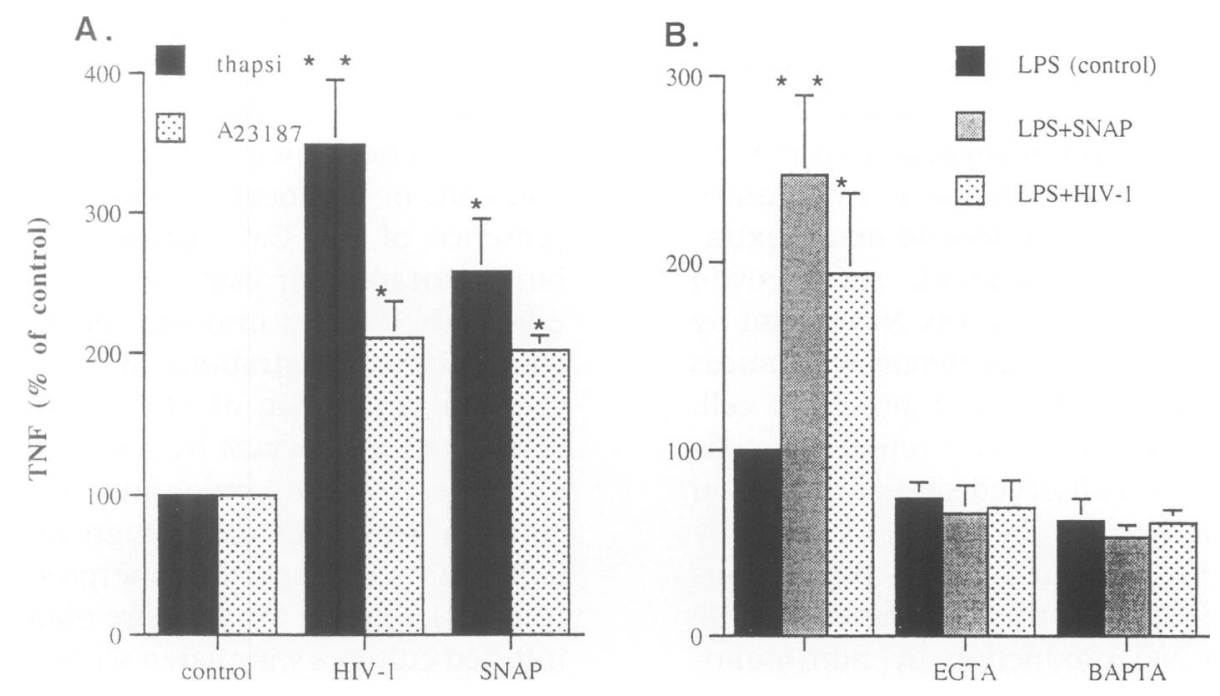

FIG. 4. NO-mediated amplification of cytokine production is $\mathrm{Ca}^{2+}$ dependent

(A) Stimulatory effect of $\mathrm{Ca}^{2+}$ inducers is amplified by HIV-1 and NO. HIV-1-infected and replicate uninfected monocyte cultures were treated with $\mathrm{Ca}^{2+}$ inducers thapsigargin (thapsi, $0.1 \mu \mathrm{M}$ ) and A23187 (2 $\left.\mu \mathrm{M}\right)$. To substitute for HIV-specific NO, uninfected cultures were also treated with SNAP (100 $\mu \mathrm{M})$. TNF levels were assayed by ELISA $18 \mathrm{hr}$ after stimulation. Values are scaled relative to uninfected cultures treated with thapsigargin and A23187 alone (control, 100\%). (B) Chelators of $\mathrm{Ca}^{2+}$ eliminate stimulatory effects of HIV-1 and NO on LPS-induced cytokine production. HIV-1-infected and uninfected (untreated [control] or treated with $100 \mu \mathrm{M}$ SNAP) monocyte cultures were treated with $0.5 \mathrm{ng} / \mathrm{ml}$ LPS in combination with $\mathrm{Ca}^{2+}$ chelators EGTA ( $\left.3 \mathrm{mM}\right)$ and BAPTA $(3 \mathrm{mM})$. Eighteen hours after stimulation, TNF levels were assayed in the supernatants by ELISA, and results were scaled relative to controls (100\%). Error bars for Panels A and B represent SD from a representative experiment (out of two performed with cells from different donors) done in triplicate (i.e., in three independent wells), and statistical analysis was performed as in Fig. 1.

HIV-1 infection. Surprisingly, presence of L-NMMA in the culture medium had only a minor (less than $20 \%$ ) effect on HIV-1 replication, and this effect was inhibitory rather than enhancing (results not shown). This result is counterintuitive, but we think the explanation for this lack of anti-viral activity of NO lies in the low levels of this factor produced by HIV-infected monocyte cultures. These levels are insufficient to exert any direct anti-viral effect but are adequate to affect the state of cell activation, thus indirectly enhancing HIV-1 replication.

\section{DISCUSSION}

In this paper, we demonstrated a critical role of NO in establishing an abnormal hyperactivated phenotype of HIV-1-infected monocytes. Our recent work (10) has shown elevated levels of NOS RNA and NO in HIV-l-infected monocyte cultures. This was an unexpected discovery, since human monocytes, in contrast to their murine counterparts, do not normally respond to activation or infection by substantially increasing NO production $(17,30)$. Even these unusual induced levels of NO production by HIV-infected human monocytes were about 10 times lower than those typically achieved by murine macrophages stimulated with interferon- $\gamma$ and LPS (31). These lower levels probably cannot account for pathological effects on bystander cells, in the manner shown for NO-mediated neurotoxicity in mice (32), so this mechanism of direct NO-mediated toxicity seems unlikely in HIV-1 infection. However, as we show in this paper, intracellular concentrations of NO in HIV-1-infected monocytes are sufficient to amplify the stimulatory effects of low doses of LPS. Since opportunistic infections are common in HIV-1-infected patients, the twosignal mechanism (i.e., mediated by LPS and NO) of hyperactivation of HIV-infected macrophages would appear likely.

Our results demonstrate that NO can stimulate monocytes by amplifying the calcium signals, thus resembling the synergism between NO and $\mathrm{Ca}^{2+}$ in the activation of immediate-early genes in neuronal cells (14). This suggests that a similar cooperation between HIV-induced NO and LPS-evoked calcium elevation might even- 
tually lead to the enhanced expression of the cytokine genes. In contrast to neuronal cells (14), however, NO effects in monocytes are cGMP-independent. A similar cGMP-independent activation of human PBMC by NO-generating compounds was described by Lander et al. (13). The signal transduction pathways linking NO to calcium-mediated activation of TNF expression in HIV-infected monocytes are the subject of ongoing studies in our laboratories.

\section{ACKNOWLEDGMENTS}

The authors wish to thank K. Manogue for critical reading of the manuscript and helpful comments, and A. Cerami for continued encouragement and support. This work was supported in part by the AmFAR Grant 02059-15-RGR (MB) and by funds from The Picower Institute for Medical Research.

\section{REFERENCES}

1. Gurram M, Chirmule N, Wang XP, Ponugoti N, Pahwa S. (1994) Increased spontaneous secretion of interleukin 6 and tumor necrosis factor alpha by peripheral blood lymphocytes of human immunodeficiency virus-infected children. Pediatr. Infect. Dis. J. 13: 496501.

2. Emilie D, Fior R, Jarrousse B, et al. (1994) Cytokines in HIV infection. Int. J. Immunopharmacol. 16: 391-396.

3. Clouse KA, Powell D, Washington I, et al. (1989) Monokine regulation of human immunodeficiency virus-1 expression in a chronically infected human $\mathrm{T}$ cell clone. J. Immunol. 142: 431-438.

4. Poli G, Fauci AS. (1992) The role of monocyte/macrophages and cytokines in the pathogenesis of HIV infection. Pathobiology 60: $246-251$.

5. Poli G, Fauci AS. (1993) Cytokine modulation of HIV expression. Semin. Immunol. 5: 165-173.

6. Tyor WR, Glass JD, Griffin JW. (1992) Cytokine expression in the brain during the acquired immunodeficiency syndrome. Ann. Neurol. 31: 349-360.

7. Wesselingh SL, Power C, Glass JD, et al. (1993) Intracerebral cytokine messenger RNA expression in acquired immunodefi- ciency syndrome dementia. Ann. Neurol. 33: 576-582.

8. Schmidtmayerova H, Nottet HSLM, Nuovo $G$, et al. (1996) HIV-1 infection alters chemokine $\beta$ peptide expression in human monocytes: implications for recruitment of leukocytes into brain and lymph nodes. Proc. Natl. Acad. Sci. U.S.A. 93: 700-704.

9. Nottet HSLM, Jett $M$, Flanagan $C R$, et al. (1995) A regulatory role of astrocytes in HIV-1 encephalitis. An overexpression of eicosanoids, platelet-activating factor, and tumor necrosis factor- $\alpha$ by activated HIV-1infected monocytes is attenuated by primary human astrocytes. J. Immunol. 154: 35673581 .

10. Bukrinsky MI, Nottet HS, Schmidtmayerova $\mathrm{H}$, et al. (1995) Regulation of nitric oxide synthase activity in human immunodeficiency virus type 1 (HIV-1)-infected monocytes: implications for HIV-associated neurological disease. J. Exp. Med. 181: 735-745.

11. Bredt DS, Snyder SH. (1994) Nitric oxide: a physiologic messenger molecule. Annu. Rev. Biochem. 63: 175-195.

12. Lander HM, Sehajpal PK, Novogrodsky A. (1993) Nitric oxide signaling: A possible role for G proteins. J. Immunol. 151: 7182-7187.

13. Lander HM, Sehajpal P, Levine DM, Novogrodsky A. (1993) Activation of human peripheral blood mononuclear cells by nitric oxide-generating compounds. J. Immunol. 150: 1509-1516.

14. Peunova N, Enikolopov G. (1993) Amplification of calcium-induced gene transcription by nitric oxide in neuronal cells. Nature 364: $450-453$.

15. Stamler JS. (1995) Redox signaling: Nitrosylation and related target interactions of nitric oxide. Cell 78: 931-936.

16. Zinetti M, Fantuzzi G, Delgado R, Di Santo E, Ghezzi P, Fratelli M. (1995) Endogenous nitric oxide production by human monocytic cells regulates LPS-induced TNF production. Eur. Cytokine Netw. 6: 45-48.

17. Padgett EL, Pruett SB. (1992) Evaluation of nitrite production by human monocytederived macrophages. Biochem. Biophys. Res. Commun. 186: 775-781.

18. Moncada S, Palmer RMJ, Higgs EA. (1991) Nitric oxide: Physiology, pathophysiology, and pharmacology. Pharmacol. Rev. 43: 109142.

19. Lancaster JR. (1994) Simulation of the diffusion and reaction of endogenously pro- 
duced nitric oxide. Proc. Natl. Acad. Sci. U.S.A. 91: 8137-8141.

20. Stuehr DJ, Nathan CF. (1989) Nitric oxide. A macrophage product responsible for cytostasis and respiratory inhibition in tumor target cells. J. Exp. Med. 169: 1543-1555.

21. Mannick JB, Asano K, Izumi K, Kieff E, Stamler JS. (1994) Nitric oxide produced by human B lymphocytes inhibits apoptosis and Epstein-Barr virus reactivation. Cell 79: 1137-1146.

22. Lee CG, Demarquoy J, Jackson MJ, O'Brien WE. (1994) Molecular cloning and characterization of a murine LPS-inducible cDNA. J. Immunol. 152: 5758-5767.

23. Letari $O$, Nicosia $S$, Chiavaroli $C$, Vacher $P$, Schlegel W. (1991) Activation by bacterial lipopolysaccharide causes changes in the cytosolic free calcium concentration in single peritoneal macrophages. J. Immunol. 147: 980-983.

24. Thastrup O, Cullen PJ, Drobak BK, Hanley MR, Dawson AP. (1990) Thapsigargin, a tumor promoter, discharges intracellular $\mathrm{Ca}^{2+}$ stores by specific inhibition of the endoplasmic reticulum $\mathrm{Ca}^{2+}$-ATPase. Proc. Natl. Acad. Sci. U.S.A. 87: 2466-2470.

25. Hurme $M$, Viherluoto J, Nordstrom $T$. (1992) The effect of calcium mobilization on LPS-induced IL- $1 \beta$ production depends on the differentiation stage of the monocytes/ macrophages. Scand. J. Immunol. 36: 506511.

26. Mulsch A, Luckhoff A, Pohl U, Busse R, Bassenge E. (1989) LY-83583 (6-amilino-5,8quinolinedione) blocks nitrovasodilator-in- duced cyclic GMP increases and inhibition of platelet activation. Naunyn Schmiedeberg's Arch. Pharmacol. 340: 119-125.

27. Croen KD. (1993) Evidence for an antiviral effect of nitric oxide. Inhibition of herpes simplex virus type 1 replication. J. Clin. Invest. 91: 2446-2452.

28. Karupiah G, Xie QW, Buller RM, Nathan C, Duarte C, MacMicking JD. (1993) Inhibition of viral replication by interferon-gamma-induced nitric oxide synthase. Science 261: 1445-1448.

29. Akarid K, Sinet M, Desforges B, GougerotPocidalo MA. (1995) Inhibitory effect of nitric oxide on the replication of a murine retrovirus in vitro and in vivo. J. Virol. 69: 7001-7005.

30. Schneemann M, Schoedon G, Hofer S, Blau N, Guerrero L, Schaffner A. (1993) Nitric oxide synthase is not a constituent of the antimicrobial armature of human mononuclear phagocytes. J. Infect. Dis. 167: 13581363.

31. Lorsbach RB, Murphy WJ, Lowenstein CJ, Snyder SH, Russell SW. (1993) Expression of the nitric oxide synthase gene in mouse macrophages activated for tumor cell killing. Molecular basis for the synergy between interferon-gamma and lipopolysaccharide. J. Biol. Chem. 268: 1908-1913.

32. Dawson VL, Dawson TM, Bartley DA, Uhl GR, Snyder SH. (1993) Mechanisms of nitric-oxide-mediated neurotoxicity in primary brain cultures. J. Neurosci. 13: 26512661. 